\title{
Particle Physics with DIS - on the European Particle Physics Strategy Update
}

\section{Néstor Armesto*}

Universidade de Santiago de Compostela, Departamento de Física de Partículas and IGFAE, 15782 Santiago de Compostela, Galicia-Spain

E-mail: nestor.armesto@usc.es

\section{Allen Caldwell*}

Max Planck Institute for Physics, Munich, Germany

E-mail: caldwell@mppmu.mpg.de

\section{David d'Enterria*}

CERN, European Organization for Nuclear Research, Geneva, Switzerland

E-mail: david.d'enterria@cern.ch

\section{Yulia Furletova}

Jefferson Lab, Newport News, VA 23606, USA

E-mail: yulialjlab.org

\section{Max Klein*}

University of Liverpool, Department of Physics, L697ZE Liverpool, Oxford Street, United Kingdom

E-mail: mklein@hep.ph.liv.ac.uk

\author{
Aharon Levy* \\ Tel Aviv University, Raymond \& Beverly Sackler Faculty of Exact Sciences, Chaim Levanon St \\ 55, Tel Aviv-Yafo, Israel \\ E-mail: aharon. levy@cern.ch \\ Jian-Wei Qiu* \\ Jefferson Lab, Theory Center, 12000 Jefferson Avenue, Newport News, VA 23606, USA \\ E-mail: jqiu@jlab.org
}

\section{Christian Schwanenberger}

Deutsches Elektronen-Synchrotron, Notkestr. 85, 22769 Hamburg, Germany

E-mail: christian.schwanenbergerecern.ch

\section{Bernd Surrow}

Temple University, Department of Physics, 1925 N. 12th St., Philadelphia, PA 19122, USA

E-mail: surrowetemple.edu

\section{Bo-Wen Xiao}

Central China Normal University, Key Laboratory of Quark and Lepton Physics (MOE) and Institute of Particle Physics, Wuhan 430079, China

E-mail: bxiao@mail.ccnu.edu.cn 
This document sketches the physics perspectives and project status of various novel electronhadron collision facilities proposed for deep inelastic scattering studies beyond HERA, including electron-ion colliders in the US (EIC) and China (EicC), energy-frontier machines at very high luminosity (LHeC and FCCeh) at CERN, and the VHEep project based on novel plasma electron accelerator technology at CERN. This summary is based mainly on presentations and discussions at a panel on the future of European particle physics, held at Kobe (Japan) during the 2018 DIS conference. The contents of this report will be included in the DIS-community recommendations to the EPPSU document to be updated by May 2020.

XXVI International Workshop on Deep-Inelastic Scattering and Related Subjects

16-20 April 2018

Kobe University Convention Centre / Kobe International Conference Center, Kobe, Japan

* Speaker. 


\section{Electron-Hadron Scattering in the Strategy Process 2013 and Now}

In the final document of European Particle Physics Strategy Update (EPPSU) of 2013 [1], there was no direct mention of future electron-hadron scattering experiments nor on studies of QCD and the proton structure. The situation in 2018 has evolved; the high-luminosity LHC (HL-LHC) has become an approved project, and the expectation is for the LHC to operate until close to 2040; CERN has created the Future Circular Collider (FCC) study [2] integrating ee [3], hh [4], and eh [5] collider options, and it has issued a mandate to develop the LHeC [6], an energy recovery linac to possibly be coupled with the LHC, and its successors, the high-energy LHC (HE-LHC, with twice the LHC c.m. energy) or/and the FCC. As it was the case in 2013, there is still no decision about the Japanese ILC, while three other $e^{+} e^{-}$proposals (CLIC, CepC, and FCC-ee) advanced. CERN has created the neutrino platform which basically supports the development of DUNE at Fermilab.

Both the context of the future of particle physics, driven by the physics of the LHC, and the design of ep/eA scattering projects have undergone a lot of developments in the past five years:

i) the $\mathrm{LHeC}$ has been developed towards a high-luminosity $\left(10^{34} \mathrm{~cm}^{-2} \mathrm{~s}^{-1}\right)$ facility for QCD, new physics searches, and Higgs studies at $\mathrm{TeV}$ energies to complement the LHC, the highenergy option of LHC (HE-LHC) and possibly the FCC, advancing also the new technology of energy recovery and superconducting RF, in line with the 2013 strategy;

ii) in the US, a high-luminosity lower-energy project has advanced, the EIC, for spin, hadron and nuclear physics through electron-hadron scattering, with emphasis on polarized proton and nuclear beams, respectively, which will use energy recovery techniques, too;

iii) a long-term perspective was recognized in the potential of generating high energy, above a $\mathrm{TeV}$, electron beams through novel plasma wakefield technology.

The following is a report about evaluations and discussions of these projects, still under design, and perspectives, including theoretical advances, which took place at the DIS Workshop at Kobe (Japan), in April 2018, where the participants agreed on the importance of building novel electronhadron colliders, following HERA, to advance nuclear and particle physics.

\section{The update process}

On Tuesday April 17th 2018, on the second day of the XXVI International Workshop on Deep Inelastic Scattering and Related Subjects (DIS 2018), a special panel discussion was dedicated to the upcoming EPPSU. It was attended by about 260 participants.

The panel discussion consisted of the following research fields:

- Electron Ion Collider (EIC) physics [7]

- The Large Hadron electron Collider (LHeC) [8]

- The Very High Energy electron proton collider (VHEep) [9]

- Future Circular Colliders (FCC) [10] 
- QCD theory studies [11]

- Heavy ion physics [12].

The discussion also included contributions, comments, and questions from the audience.

The goal of the panel discussion was to show that all the different electron-hadron projects have many common subjects and complement each other. The present document will be used as input to the document that will be submitted to the EPPSU as the recommendation of the DIS community at the end of 2018 .

\section{Proposed future DIS facilities}

The LHC is the only accelerator presently providing high-energy proton and ion beams, being the only machine that can provide hadron-beams for DIS at the energy frontier in the next two decades. The mission of ep/eA colliders is to address fundamental questions in strong interaction/QCD physics by exploring the substructure and dynamics of matter at scales relevant for collider energies, and to carry out high-precision QCD, Higgs, top and electroweak measurements, as well as to search for new phenomena. This will uniquely improve our understanding of the QCD and electroweak sectors of the Standard Model (SM) to new qualitative and quantitative levels and allow the exploration of physics beyond the Standard Model (BSM) with the highest sensitivity. The specifics of DIS is the spacelike interaction configuration and the pointlike probe of hadrons. With respect to $h h$, DIS is a clean process with neutral- and charged-current separation and no pile-up, and it extends to higher collision energies, above a TeV.

In addition, DIS facilities can distinguish themselves from $h h$ colliders by measuring deeply virtual Compton scatterings which keep the colliding $h$ intact even at hard collisions with pointlike probes. This makes them ideal and unique for exploring the tomographic imaging of the confined quark-gluon landscape [13] inside the hadron.

As with the exploration of the Fermi scale with Tevatron, LEP/SLC, and HERA, the aim is now to study physics at the TeV scale with $h h$, ee and $e h$ colliders. Specifically, DIS is giving clean access, through large virtualities $(Q)$ and very low Bjorken $x$, to the smallest dimensions, to new phenomena and the Higgs (SM and BSM) properties with electron-hadron interactions, complementary to $e^{+} e^{-}$and $h h$. The high-energy frontier DIS is underpinned by lower-energy nuclear DIS, which provides yet a further important complementarity to the future development of high-energy physics. There are several proposals to build electron-hadron colliders worldwide. Various facilities under consideration are listed with some key parameters summarized in Table 1.

The rich nuclear and particle physics programs of these collider projects were discussed by six panel members and are summarized in the following.

\subsection{The Electron Ion Collider (EIC)}

The US based EIC [14] has two facility concepts, namely JLEIC and eRHIC, which are based on the existing CEBAF facility at the Jefferson Laboratory and RHIC machine at Brookhaven National Laboratory, respectively. In addition, there has been a proposal to build an Electron Ion Collider (EIC) in China (EicC) with similar accelerator parameters but slightly lower energy. The EIC is proposed to be a high-luminosity and high-precision machine with both polarized electron 
Table 1: Overview of proposed electron-hadron colliders.

\begin{tabular}{llllll}
\hline \hline Facility & Years & $\begin{array}{l}E_{c m} \\
(\mathrm{GeV})\end{array}$ & $\begin{array}{l}\text { Luminosity } \\
\left(10^{33} \mathrm{~cm}^{-2} \mathrm{~s}^{-1}\right)\end{array}$ & Ions & Polarization \\
\hline EIC in US [14] & $>2028$ & $20-100 \rightarrow 140$ & $2-30$ & $\mathrm{p} \rightarrow \mathrm{U}$ & $e, \mathrm{p}, \mathrm{d},{ }^{3} \mathrm{He}, \mathrm{Li}$ \\
EIC in China [15] & $>2028$ & $16 \rightarrow 34$ & $4 \rightarrow 100$ & $\mathrm{p} \rightarrow \mathrm{Pb}$ & $e, \mathrm{p}$ and light nuclei \\
LHeC (HE-LHeC) [6] & $>2030(2044)$ & $200 \rightarrow 1300(1800)$ & 10 & $\mathrm{p} \rightarrow \mathrm{Pb}$ & e possible \\
PEPIC [16] & $>2025-2030$ & $530 \rightarrow 1400$ & $<10^{-3}$ & depends on LHC & depends on source \\
VHEep [16] & $>2038$ & 9000 & $10^{-5}-10^{-4}$ & depends on LHC & depends on source \\
FCC-eh [5] & $>2044$ & 3500 & 15 & depends on FCC & e possible \\
\hline \hline
\end{tabular}

and hadron (proton and light ions) beams as well as the capability of accelerating various types of heavy ions. The EIC can measure many different types of transverse momentum dependent distributions (TMD) and generalized parton distributions (GPD) on top of precise measurements of collinear parton distribution functions (PDF), thereby leading to multi-dimensional tomography of hadrons. It is also of great importance to fully understand the origin of proton spin and to study various spin effects at the EIC. The physics mission also includes nucleon-nucleon correlations inside heavy nuclei, emergence and propagation of hadrons in the cold nuclear medium, as well as probing partons in the non-linear regime of QCD (parton saturation). The ultimate objective of the EIC projects is to understand QCD interactions and emergence of hadronic and nuclear matter in terms of quark and gluon degrees of freedom, through the synergy of experimental and theoretical progress.

In the following, we address the EPPSU calls regarding the EIC:

- Scientific context: High-energy hadron and nuclear physics and spin physics with strong implications on SM physics.

- Objectives: Address fundamental questions on the structure and dynamics of nucleons and nuclei in terms of quarks and gluons using precision measurements.

- Methodology: Measurements of inclusive, semi-inclusive, and exclusive DIS processes including scatterings with polarized beams and various ions.

- Readiness: For the US-EIC, it has been reviewed by the US National Academy of Sciences (NAS), and the final NAS report was recently published [17]. CD-0 (US Mission Need Statement for the Critical Decision) could be awarded in 2018/2019. For the EicC, the white paper is planned to be delivered by 2019 .

- Expected Challenges: various (critical) accelerator R\&D questions will not be answered until 2019. The selection of the site may occur around 2019/2020. The EIC facility construction has to start after the completion of FRIB (Facility for Rare Isotope Beams), construction to ramp down around 2020. The best guess for the earliest date for completion of the EIC facility construction would be 2028-2030 — in roughly a decade from now.

\subsection{Large Hadron electron Collider (LHeC)}

The $\mathrm{LHeC}$ is an accelerator proposal [6] for the high-luminosity upgrade of the LHC (HLLHC) by adding a $60 \mathrm{GeV}$ electron energy recovery linac to the current LHC proton (heavy ion) 
accelerator ring. It can provide electron-proton and electron-ion collisions running concurrently to the LHC operation at unprecedented high energies $(\sqrt{s} \sim 200-1300 \mathrm{GeV})$ and high luminosities $\left(10^{34} \mathrm{~cm}^{-2} \mathrm{~s}^{-1}\right)$, which lead to high-precision results.

The raison d'être of the $\mathrm{LHeC}$ can be cast into the following goals: firstly, it is a "giant microscope" with highest and cleanest resolution with the capability to probe QCD matter substructures at the smallest scale. Secondly, the clean $e h$ DIS process allows to maximize the utility of the LHC by providing reliable inputs and reducing uncertainties (such as for PDFs and the QCD coupling $\alpha_{s}$ ) which will lead to a higher reach to discover new particles. Thirdly, it transforms the LHC into a high-precision Higgs facility. Lastly, it is a unique hadron and nuclear physics facility which can study non-linear QCD evolution, solving the question of gluon saturation, diffractive scattering, small- $x$ physics in protons and nuclei, and nuclear PDFs in a wide range of kinematical phase space, but also has a unique and rich electroweak physics, top physics, Higgs physics, and BSM physics program.

In the following, we address the EPPSU calls regarding the LHeC:

- Scientific context: Dynamics and discovery in QCD, SM and exotic Higgs Physics, BSM.

- Objectives: Discovery of new QCD dynamics (BFKL, saturation, instantons), discovery of new physics in Higgs, SUSY, top, Dark Matter and heavy neutrino sectors. Reconstruction of proton and nuclear structure in full and deeper than hitherto. Constraint of SM with hugely increased precision, search for unified theory. Clarification of cold nuclear matter baselines for quark gluon plasma studies. 3D proton structure.

- Methodology: High-precision measurements of inclusive and semi-inclusive DIS and photoproduction processes (including electron-ion scattering with seminal extension of kinematic range), and vector-boson-fusion Higgs production.

- Readiness: Accelerator: needs technology development ERL facility for accompanying LHeC development (PERLE). Main step: ERL multi-turn demonstration - expected in 2020. Detector: compared to $p p$ scattering at the LHC the radiation level is $1 / 100$ lower, the pileup 0.1 instead of 150 . There is a design exploiting known technology, which can be realized without R\&D by a Collaboration being formed.

- Expected Challenges: High current multi-turn ERL and Design of IR. For detector: forward tracking and calorimetry.

\subsection{Future Circular Collider (FCC)}

The FCC [2] configuration is based on a $100 \mathrm{~km}$ circumference tunnel housing initially a highluminosity $e^{+} e^{-}$collider of $90-350 \mathrm{GeV}$ cms energies [3] followed by a $p p$ collider of $100 \mathrm{TeV}$ cms energy [4]. The FCC-eh [5] is largely defined through the FCC-hh, complemented by an electron accelerator, chosen to be the ERL of the $\mathrm{LHeC}$, but positioned at point $\mathrm{L}$ of the FCC ring. As for the LHC, $e p$ and $p p$ are designed to operate concurrently such that during the lifetime of the FCC about $2 a b^{-1}$ of integrated luminosity can be collected for the $e h$ option. The Higgs production cross section at the FCC-eh is about $1 \mathrm{pb}$, such that rougly the same number of Higgs bosons is produced in $e p$ as in $e^{+} e^{-}$collisions at FCC-ee taking into account the different luminosity and 
operation projections. This, complemented by the rare process sensitivity in $p p$, provides an unprecedented laboratory for sub-percent precision Higgs measurements jointly with $e^{+} e^{-}$, including the exploration of the Higgs field potential via double-Higgs production. The FCC-eh extends to Bjorken $x$ of $10^{-7}$ (and 1) where new parton dynamics becomes relevant and is to be established for understanding $p p$ physics at the FCC. The FCC, including he, is a phenomenal vision for the development of high-energy physics in our century.

In the following, we address the EPPSU calls regarding the FCC:

- Scientific context: Search for new physics and high-precision tests of SM physics including precision Higgs and top physics as well as high-energy nuclear physics.

- Objectives: Direct/indirect BSM searches and discovery up to $50 \mathrm{TeV}$, and unparalleled QCD research potential.

- Methodology: All processes in $e^{+} e^{-}, e p$ and $p p$ collisions.

- Readiness: The conceptual design is planned to be delivered before the end of 2018, in time for the EPPSU.

- Expected Challenges: High field dipole magnet development and production, large-scale civil engineering, cost reduction.

\subsection{Very High Energy electron proton (VHEep) accelerator}

VHEep [16] is a result of an international community wide development of a novel accelerator mainly based on proton driven plasma wake field technology, which can accelerate electrons to very high energy (around $3 \mathrm{TeV}$ ) using the LHC driver. A similar version, which is known as PEPIC as a demonstration project for VHEep, uses SPS driver to accelerate electrons. Essentially, it leads to an ultra high energy but low-luminosity collider, which can be useful for high-energy physics which does not require high luminosities. Its targeted physics objectives are studies of QCD dynamics at extremely small- $x$ and gluon saturation, the measurement of vector meson production and the exploration of the high-energy limit of total cross-sections as well as selected opportunities in the search for BSM physics.

In the following, we address the EPPSU calls regarding the VHEep:

- Scientific context: High-energy low- $x$ physics with opportunities in BSM searches.

- Objectives: Understand ultra high-energy behavior of ep collisions.

- Methodology: Measurement DIS processes with large cross sections.

- Readiness: At the start of the technology demonstration.

- Expected Challenges: Technology demonstration by 2024 with determined luminosity, electron energies, etc. 


\section{Theory Input for Future DIS Machines}

Future DIS machines allow the study of a very rich and diverse physics program. This includes the investigation of the structure of the nucleon, parton distributions, spin physics, QCD and high density dynamics in the nucleon and nuclear matter. It also includes high precision electroweak, top quark, and Higgs boson properties measurements, and sensitive searches for physics beyond the SM.

In particular, the role of QCD and that of Heavy Ion theories were discussed by two members of the panel as outlined in the following subsections. At the panel, theory developments on Higgs, BSM and electroweak physics were not covered. A description of the theory motivation in addition to QCD is important as well since future DIS machines will also perform a rich physics program beyond QCD. Furthermore, it was mentioned during the discussion that the acceptance of projects such as the $\mathrm{LHeC}$ by the particle physics community needs to rely on various types of physics involving apart from novel QCD measurements also electoweak, top, Higgs and BSM physics.

\subsection{Dual Role of QCD theory}

QCD has been extremely successful in interpreting the vast amount of data collected from all high-energy hadronic scattering experiments, in particular thanks to the perturbative QCD factorization theorem, which combines short distance hard parts, theoretically calculable, with universal PDFs, extractable from high-energy $e p$ and $p p$ collisions. Any new physics searches based on hadron-hadron or electron-hadron colliders rely heavily on PDFs. The best way to gain precise information about PDFs is to measure them in an electron-hadron collider at high energy and high luminosity. QCD represents the fundamental theory which describes the strong force binding quarks and gluons and eventually almost all the visible matter making up the visible world. New theory advancements in recent years, in extending the perturbative QCD factorization theorem to observables with multiple physical scales, made it possible to explore the confined motion and spatial distributions of quarks and gluons inside the hadron with pointlike probes at high-energy/luminosity eh DIS facilities.

Currently, the new physics search at the LHC is limited by large uncertainties in partonic flux at large momentum scales. Precision electroweak, Higgs, and top studies will be also ultimately hindered by PDF and $\alpha_{s}$ uncertainties. It is very important to have good reach in large $x$ and $Q^{2}$ regions in DIS which allows to improve our knowledge of PDFs in a much wider $x, Q^{2}$ range. Furthermore, it is also of fundamental importance to study QCD in order to probe different limits of the QCD evolution, and to understand the spin and 3D structure of hadrons in terms of quark and gluon degrees of freedom. Ultimately, one can depict the colorful multi-dimensional landscape of the internal structure of hadrons, including nucleons and heavy nuclei, providing much needed information and hints on the confined nature of quarks and gluons, and their correlations. In addition, one can study the color interactions between nucleons to explore the origin of nuclear force and the emergent phenomena of QCD in $e A$ collisions by using nuclei as femtometer-sized detectors.

\subsection{Heavy Ion Physics}

It has been widely accepted that deconfined quark gluon plasma has been created in heavy ion collisions at high energies at RHIC and the LHC. Two main features of the heavy ion data 
indicate that (i) such matter shows strong collectivity in the soft sector that is well described by relativistic hydrodynamics if applied very early (at times smaller than $1 \mathrm{fm} / \mathrm{c}$ ) after the collision; (ii) it is extremely opaque to energetic partons or particles traversing it, as evident from the strong modification of the yield of hard probes like heavy colored bound-states, high- $p_{T}$ jets, etc.

DIS on proton and heavy ion targets will contribute to answer some most fundamental questions in nuclear and heavy ion physics, such as:

- provide a detailed picture of the initial wave function of protons and nuclei, in terms of both longitudinal and transverse partonic densities;

- clarify the mechanism of particle production, including a possible breaking of factorization in some regions of phase space, via a comparison of DIS, $p p, p A, A A$ results with theoretical calculations with accurate parton densities;

- serve as a clean benchmark to understand the mechanism of isotropization/thermalization of partonic matter that allows the use of hydrodynamics calculations to reproduce the data, by studying how small a system can become and still show collective features;

- characterize accurately the cold nuclear matter effects impacting the production cross sections of perturbative processes, hard probes, used for medium "tomographic" analysis in heavy ion collisions. Note that any step in eA collider physics is expected to lead to progress since the range and systematics in fixed target lepton-hadron experiments are inferior to a collider configuration of significant energy and luminosity, and especially because HERA did not study electron-ion physics.

In order to achieve these goals, both $e p$ and $e A$ are required for the search for non-linear QCD evolution and a systematic study of the difference between nuclear PDFs and proton PDFs. Extended reach in collision energies will also make it possible to have a sizeable lever arm in $Q^{2}$ at small $x$ to study saturation, and to explore rarer hard probes (top quarks, Higgs bosons) that will play a role in heavy ion physics at future colliders such as the HE-LHC or FCC.

Electron-ion scatterings will be complementary to the $p A$ and ultraperipheral programs at hadron colliders, which have larger kinematic reach but are less precise both from the experimental point of view - full kinematic reconstruction is not possible in hadronic colliders - and from the theoretical point of view - the accuracy in perturbative calculations is much larger for inclusive DIS than for exclusive processes in photoproduction or semi-inclusive ones in $p A$. The EIC will partially overlap with the kinematic region previously measured in fixed target experiments and partially with the HERA kinematic region. The focus is on high-precision measurements including varying nuclear species and particle identification down to low transverse momenta, as well as polarized beams being a versatile machine. The $\mathrm{LHeC}$ and FCC-eh, running concurrently with the respective $h h$ or $A A$ programs, will cover a much larger kinematic region, with access to very small $x$ and very large $Q^{2}$ of relevance for future $h h / A A$ colliders. This, together with the different additional rich physics programs of the EIC and LHeC/FCC-eh machines which complement each other, makes them crucial for the interpretation of the results in heavy ion collisions. 


\section{The European Particle Physics Strategy Update 2018}

As mentioned above, it is absolutely vital to have lepton-hadron-scattering machines in the near future. The rich program involves high-energy nuclear physics and high-energy tests of the SM of particle physics, and sensitive searches for physics beyond it. The physics program is competitive and complementary to other future collider projects involving $e^{+} e^{-}$and $h h$ collisions.

The three lines of development may be briefly summarised as follows.

- A rich and interesting science program in hadron and nuclear physics of a high-luminosity DIS machine. At the EIC, besides an intriguing physics program in spin physics, it is also interesting to study QCD evolution in extreme conditions of $x$ and $Q^{2}$, and fully describe the 3D structure of hadrons in terms of quark and gluon degrees of freedom. DIS on heavy ion targets can also provide information on the mechanism of in-medium and vacuum hadronization, and the emergent phenomena of QCD.

- A rich and diverse science program in particle physics of a high-energy DIS machine. The $\mathrm{LHeC}$, and later the FCC-eh, allow a unique research program on the fields of high-energy QCD, non-linear QCD phenomena, diffraction, hadronic and nuclear physics, high-precision top and electroweak measurements and sensitive searches for phenomena beyond the SM. Together with the high-precision measurements possible in Higgs physics, the LHeC is an attractive project running in parallel and complementing the HL-LHC.

- Plasma acceleration. The AWAKE project should demonstrate within the next years that electrons can be accelerated with a high gradient and good emittance in a plasma in the wake of proton bunches. A colliding beam experiment that has been envisaged may operate concurrently with the LHC through eh collisions using the SPS at CERN (PEPIC). Its energy range would extend the one of HERA. A longer-term project based on the same technology represents the ep/eA collider VHEeP that can analyse the fundamental structure of hadronic matter at the $10 \mathrm{TeV}$ scale.

\section{References}

[1] M. Krammer, Phys. Scripta T 158, 014019 (2013).

[2] The Future Circular Collider, a Conceptual Design Report, CERN, Geneva 2017 and 2018, in preparation.

[3] M. Bicer et al. [TLEP Design Study Working Group], JHEP 1401, 164 (2014); D. d'Enterria, arXiv: 1602.05043 [hep-ex].

[4] M. Mangano, arXiv:1710.06353 [hep-ph].

[5] M. Klein, Ann. Phys. (Berlin) 528, No. 1âĂŞ2, 138 (2016).

[6] J. L. Abelleira Fernandez et al. [LHeC Study Group], J. Phys. G 39, 075001 (2012).

[7] B. Surrow, "The case for EIC", in these proceedings, 2018,

https://indico.cern.ch/event/656250/contributions/2939330/

attachments/1634134/2612093/bsurrow-eic-case-tuesdsay-dis2018.pdf. 
[8] M. Klein, "The case for LHeC", in these proceedings, 2018, https://indico.cern.ch/event/656250/contributions/2939331/ attachments/1634262/2606713/caseLHeCmaxklein.pdf.

[9] A.-C. Caldwell, "The case for VHEep", in these proceedings, 2018, https://indico.cern.ch/event/656250/contributions/2939332/ attachments/1634227/2606649/Panel-Caldwell.pdf.

[10] D. d'Enterria, "The case for FCC", in these proceedings, 2018, https://indico.cern.ch/event/656250/contributions/2939333/ attachments/1634240/2606674/dde_FCC_review_dis18.pdf.

[11] J. Qiu, "QCD theory addressed in the above facilities", in these proceedings, 2018, https://indico.cern.ch/event/656250/contributions/2939334/ attachments/1634189/2606577/Qiu-DIS2018-panel-discussion-QCD.pdf.

[12] N. Armesto, "Heavy Ion Theory addressed in the above facilities", in these proceedings, 2018, https://indico.cern.ch/event/656250/contributions/2939335/ attachments/1634194/2606587/armesto_DIS2018_170418.pdf.

[13] X. D. Ji, Phys. Rev. D 55, 7114 (1997).

[14] A. Accardi et al., Eur. Phys. J. A 52, 268 (2016).

[15] X.R. Chen, "Electron Ion Collider in China," in these proceedings, 2018.

[16] A. Caldwell and M. Wing, Eur. Phys. J. C 76, 463 (2016).

[17] The National Academies of Sciences, Engineering, Medicine, ISBN 978-0-309-47856-4, http: / / nap.edu/25171. 